\title{
Book review: Du leurre archéologique au milieu associé préhistorique: compte-rendu et commentaires autour de l'ouvrage La pierre et son ombre. Épistémologie de la Préhistoire d'H. Forestier
}

\author{
Antonio Pérez ${ }^{1,2}$
}

1. ArScAn-Équipe AnTET, UMR 7041, CNRS, Université Paris Nanterre (UPN), 200 Avenue de la République, 92001 Nanterre Cedex, France. Email: antonioperezbalarezo@hotmail.com

2. Institut français d'études andines, Lima, Pérou.

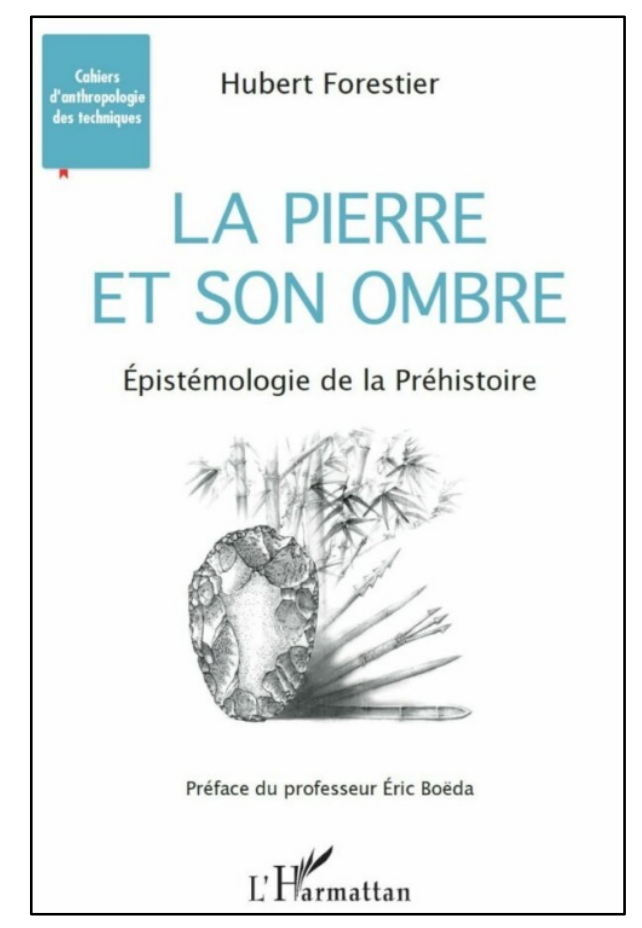

La pierre et son ombre. Épistémologie de la Préhistoire

by Hubert Forestier

L’Harmattan, 2020, pp. 272, fig. 32 ISBN 978-2-343-19641-1

https://www.editions-harmattan.fr/index.asp?navig=catalogue\&obj=livre\&no=66449

Journal of Lithic Studies (2020) vol. 7, nr. 1, 6 p.

DOI: https://doi.org/10.2218/jls.4499

Published by the School of History, Classics and Archaeology, University of Edinburgh

ISSN: 2055-0472. URL: http://journals.ed.ac.uk/lithicstudies/

Except where otherwise noted, this work is licensed under a CC BY 4.0 licence. 
Que de nos jours le titre d'un ouvrage sur la préhistoire inclue le terme " épistémologie » est pour le moins étonnant pour ne pas dire rare même si ce qualificatif est souvent associé à notre discipline, la Préhistoire. Et il faut rappeler que l'une des figures les plus reconnues de l'histoire de cette discipline, A. Leroi-Gourhan, n'était pas particulièrement encline à une réflexion épistémologique de ses propres écrits et démarches de recherche (Audouze 1999: 168). Ce fait est important si l'on considère la Préhistoire comme une discipline construite essentiellement à partir de transformations méthodologiques relativement successives dans le temps, notamment en ce qui concerne l'étude scientifique du matériel lithique archéologique. Néanmoins une question se pose: comment une discipline peut-elle supporter des rénovations méthodologiques sans changements majeurs au niveau épistémologique? Même si l'épistémologie en archéologie préhistorique a été abordée avec une certaine constance depuis les années 1960 en France et ailleurs (par exemple, de Beaune 2007; Cataldi 2016; Hussain 2019; Lowther 1962; Trigger 2007), les aspects liés à la perception scientifique du matériel lithique archéologique ont été abordés de manière tangentielle et sans une largeur et une profondeur heuristiques suffisantes.

Pour l'auteur, cette réflexion épistémologique est présentée doublement comme une excuse et comme une nécessité, du fait de la singularité du phénomène technique Hoabinhien, temporellement circonscrit entre 40000 et 10000 ans AP et spatialement dans les grottes et abrisous-roche d'Asie du Sud-Est, dont l'actuel Vietnam, Thaïlande, Laos, Cambodge et Sumatra, principalement. Une région géographique de près de 2 millions de $\mathrm{km}^{2}$. Traditionnellement défini comme un technocomplexe statique comme une " pebble culture » parmi d'autres c'està-dire sans transformations techniques majeures dans le temps et dans un contexte de forêt tropicale humide, l'Hoabinhien est essentiellement constitué d'outils façonnés unifacialement sur d'épais galets oblongs de calcaire, basalte, andésite, quartz et quartzite, appliquant la technique à la percussion directe à la pierre dure.

C'est ce phénomène technique qui interpelle l'auteur et le pousse à repenser le statut épistémologique de l'objet lithique au sein de l'archéologie préhistorique mais aussi dans le champ des capacités cognitives des Hommes Modernes en Asie du Sud-Est. Il s'agit d'une repensée, car en réalité il ne s'agit pas d'une enquête épistémologique de l'objet lithique ou de l'outil lui-même, mais plutôt d'une réflexion nouvelle de son mode de présence dans le registre archéologique, ou en utilisant les termes de l'auteur son « mode d'apparaître » au monde.

L'ouvrage est organisé en quatre grandes parties, suivies d'une conclusion. La première partie présente l'histoire disciplinaire du phénomène Hoabinhien et le décrit en fonction des approches typologiques et technologiques précédentes, ainsi que au sein de la Préhistoire de l'Asie du Sud-Est. A la suite de cette mise en contexte, l'auteur annonce le problème non évolutif de l’Hoabinhien et expose sa stratégie épistémologique et méthodologique. La seconde partie correspond à l'exposition des résultats de l'analyse techno-fonctionnelle des assemblages lithiques de l'Hoabinhien. Pour le lecteur intéressé par les détails techniques de la fabrication des outils sur galets, cette partie saura le satisfaire. La troisième partie est la dénonciation du caractère litho-centré de la préhistoire sud-asiatique et de la préhistoire universelle en général. La quatrième partie introduit pleinement le lecteur dans le milieu végétal associé de l'Hoabinhien et enfin, la conclusion expose les conséquences épistémologiques et méthodologiques de l'étude du milieu associé en Préhistoire.

Or, il s'avère que l'Hoabinhien a un mode d'apparaître, dans son écosystème de forêt tropicale humide, caractérisé par (1) une stase technique, (2) une absence de supports bordspoints ou d'objets pointus en général, et (3) une mauvaise délimitation techno-culturelle dans le contexte de la préhistoire sud-asiatique. Il est évident - ou peut-être pas du tout - qu'une approche empirico-inductiviste, classique dès le début de la discipline, permet de reconnaître ce mode d'apparaître du phénomène, mais ne permet pas de l'expliquer. Ainsi, l'auteur dénonce d'emblée un paradoxe: existe-t-il des groupes de chasseurs sans pointes et de surcroit dans les 
forêts d'Asie tropicale réputée pour ses groupes et ses modes de subsistances? Il opte donc pour une approche empirique-transcendantale, inspirée de la phénoménologie husserlienne et de la philosophie deleuzienne de la différence, qui lui permet de re-trouver une explication au-delà et en-deçà de ce mode d'apparaître sur fond d'une critique de la répétition objectale. En général, il s'agit donc d'une épistémologie délocalisée; et d'une manière particulière: une épistémologie « dé-lithocentrée » ou libérée de l’objet de pierre qui n’informe pas si ce n'est une porte de sortie. D'ailleurs, l'auteur " écrit à la fois sur l'objet lithique et à l'encontre de lui » pour mieux s’intéresser à ce qui n'est plus visible dans le champ archéologique, le végétal. Une stratégie tout à fait adéquate et une des voies possibles lorsque l'objectif est de comprendre le sens (la signification) de ce type de phénomène technique. Associée à cette épistémologie, au niveau analytique, on retrouve une méthodologie techno-fonctionnelle (Boëda 2013) qui aborde les objets lithiques au-delà de la morphologie actuelle, approfondissant leur organisation structurelle. L'application de cette méthodologie permet à l'auteur de renouveler la définition typologique de l'Hoabinhien à partir de l'identification d'une pluralité de schémas opératoires de production (uniface sur galet oblong, chopper sur galet ovoïde, outils sur split de galet), ainsi qu'une pluralité de schémas opératoires fonctionnels (unités techno-fonctionnelles transformatrices frontales, latérales, multiples, etc.; unités techno-fonctionnelles préhensives axiales et latérales; trajectoires sortantes et rentrantes; utilisation probable de sciage, coupage, fendre). Néanmoins, sur sa durée, la matrice-galet Hoabinhien de conception asymétrique ne présente toujours pas d'évolution apparente, de sorte que l'auteur y fait référence comme une " évolution buissonnière » - en contraste avec « l'évolution buissonnante » de F. Bordes - qui a probablement suivi deux trajectoires techniques: (1) apparition à l'état concret et sans évolution ultérieure; (2) apparition à l'état abstrait et stagnation presque immédiate.

Ces trajectoires et leurs lignées techniques associées sont considérées par l'auteur comme des contre-exemples de l'évolution « évidente » des techniques sur d'autres continents, rendue possible grâce à la présence d'objets plus diagnostiques - au niveau techno-culturel - que de « simples » galets. Dans le même ordre d'idées, l'auteur souligne qu'il est plus aisé d'écrire sur la préhistoire de l'Australie ou des Amériques que sur celle de l'Asie du Sud-Est, en raison de la présence de "pointes ». Nous nous permettons de signaler, à propos de la Préhistoire des Amériques, notre zone d'étude, que les premières périodes que touchent les premiers peuplements présentent également des industries à galets et à éclats qui sont oblitérés par l'omniprésence des soi-disant « pointes de projectiles ». En ce sens, si seulement nous n'avions pas autant de pointes dans les Amériques, la préhistoire américaine serait moins « cartésienne ».

Or, à propos de l'édifice épistémologique construit par l'auteur, nous nous permettons ici une digression. Dû au fait que cet édifice émerge en raison de l'impossibilité de répondre à la question comportementale classique en Préhistoire - " est-il possible de renseigner un mode de vie et une société de chasseurs sans évoquer la pointe et plus largement le projectile? » (p. 38) -, il nous semble qu'au moins partiellement le travail se poursuit sous l'ombre de cette question. Bien qu'il soit possible que cela soit dû à la nature critique (au sens étymologique du terme, i.e., $\kappa \rho i v \omega$, trier) de l'ouvrage, qui doit nécessairement précéder la construction d'un savoir phénoménologique en soi, on se demande si l'abandon depuis le début de cette question sur la réalité comportementale que l'Hoabinhien re-présente aurait contribué à atteindre plus directement l'explication de la structure technique de l'Hoabinhien. Pourquoi cet Hoabinhien et pas un autre ? On se demande si cela aurait permis non seulement de penser ou d'évoquer l'explication anthropologique, mais aussi de «tenter de l'inférer en objectivant l'objet de Pierre dans son identité techno-fonctionnelle » (p. 135). Quoi qu'il en soit, la richesse de l'ouvrage réside précisément dans cette critique épistémologique de la Préhistoire émergée du point de vue du technologue. 
Cette identité techno-fonctionnelle de la pierre est la raison du mode d'apparaître de l'Hoabinhien, un mode d'apparaître imperceptible aux sens du technologue. Il est, en langage simondonien, le milieu associé de l'objet (Simondon 2012: 69; 2013: 62) l'inverse constitutif de l'objet en tant qu'être technique individualisé, la charge pré-individuelle qui explique l'objetindividu et permet sa transindividuation, son déphasage fondamental. Notion clé pour concevoir le changement technique à partir d'une genèse technique, le milieu associé de l'objet unifacial Hoabinhien est, selon l'auteur, le végétal. En d'autres termes, l'objet technique en matière végétale est l'expression du milieu associé préhistorique associé disparu. Le végétal-nonprésent explique la pierre-présente et pour le dire à la façon de l'auteur: « le retrait du végétal expliquerait la manifestation archéologique et la nature de la pierre ». Une sorte de solidarité essentielle (une relation de causalité circulaire, d'après G. Simondon) est tissée entre ces deux modes d'existence, qui peuvent cependant s'expliquer plus universellement par le caractère préindividuel par excellence qu'est le tranchant, que ce soit sous la forme d'un végétal (bambou naturellement biseauté), ou sous une forme minérale (objet lithique naturellement biseauté). C'est pourquoi l'objet lithique est un leurre archéologique pour le technologue préhistorien - et pas seulement dans le cas de l'Hoabinhien, dans la mesure où nous sommes confrontés à des assemblages lithiques hors de notre mémoire (Boëda 2013) -, car sans la conscience de cet autre constitutif, la compréhension du phénomène technique se referme sur elle-même et il ne transcende pas sa matérialité présente. Une matérialité qui apparaît sous la forme d'une pierre doublement "seule » (la doublé solitude de la pierre, selon les termes de l'auteur): au niveau ontologique, elle n'a pas son autre transcendantal (le Végétal); au niveau épistémologique, il n'a pas son milieu associé, ce qui explique le caractère litho-centré de nos connaissances en Préhistoire. Si le préhistorien ne prend en compte que l'objet lithique, une anthropologie des techniques n'est pas viable, parce que la pierre est la re-présentation d'une "incomplétude technique » (p. 168). En partie à cause de cela, avec E. Boëda, nous avons introduit une distinction entre " milieu associé réel » et " milieu associé actuel ». Le milieu associé préhistorique, disparu, est sensu stricto un milieu associé réel. Le milieu associé actuel est ce qui survit au temps et nous parvient sous la forme d'une complémentarité techno-fonctionnelle entre les structures de production et les structures fonctionnelles, entre les nucléus/pièces bifaciales et les outils, respectivement. Dans cette perspective, l'objet lithique Hoabinhien, récupéré dans son contexte archéologique, n'est pas un individu technique, mais un artefact. L'artefact acquiert son individualité technique après une double localisation: (1) dans son contexte élémentaire or individuel, par rapport à son milieu associé; (2) dans son contexte technogénétique, par rapport à sa lignée technique. Sans considérer le milieu associé, toute production humaine sera toujours saisie à partir d'une approche instrumentale de la technique, qui conduit à la reproduction d'une asymétrie inessentielle entre l'Homme et le Milieu Naturel. Le milieu associé permet de concevoir une symétrie essentielle à la technique, où l'Homme et le Milieu Naturel coexistent au monde de la vie en termes de couplage structurel.

La démarche de $\mathrm{H}$. Forestier est originale, puissante au niveau heuristique et n'a pas fini d'interroger, car il s'agit essentiellement d'un jeu de milieux, intérieur et extérieur, qui cherche la double localisation de l'artefact. A partir d'un milieu erratique parce qu'incomplet, représenté par l'objet lithique archéologique, l'auteur cherche d'abord le milieu compréhensif, le milieu associé, le végétal, qu'il s'agisse de bambou, de rotin ou de tout autre bois dur.

Ainsi le bambou cesse de faire partie du milieu naturel (ou milieu extérieur) pour intégrer l'individualité de l'objet lithique archéologique. Le bambou est proprement l'intégration fonctionnelle (i.e., le milieu associé) d'un milieu naturel principalement non fonctionnel. En d'autres termes, la forêt participe au fonctionnement opératoire l'outil lithique à travers le bambou, son milieu associé. L'uniface Hoabinhien n'a de sens que comme pont matériel, ontologique et épistémologique, entre le milieu naturel et le bambou. L'objet lithique-lignic est, dans une large mesure, l'objet Hoabinhien. Ensuite, l'auteur cherche une explication 
phénoménologique de cette individualité hoabinhienne à partir d'une approche technogénétique, ce qui l'amène à proposer des trajectoires et des lignées techniques, détaillées auparavant.

Sur la base de tout cela, la pierre, l'objet lithique, ne veut pas dire tout en Préhistoire, propose H. Forestier. L'auteur déclare à sa façon ce que j'appellerai la «crise épistémologique » des industries lithiques. L'objet lithique n'est pas suffisant en Préhistoire, ou en tout cas dans certains phénomènes techniques préhistoriques, d'un point de vue heuristique. H. Forestier dénonce ainsi l'objectivité du fait archéologique en ouvrant la voie à la prise de conscience de sa subjectivité inhérente et aux « arrangements » intersubjectifs inévitables entre le préhistorien et l'objet de sa recherche. L'histoire de l'Hoabinhien a été racontée à partir de ce qui est montré, de ce qui apparaît: la pierre. L’auteur propose de centrer l'étude sur l'historicité de l'Hoabinhien, ce qui signifie évaluer les conditions de possibilité historique de ce qui nous est présenté. Cette " évaluation » est présentée comme un exercice fondamental avant, pendant et après l'analyse des objets lithiques. Cela implique de penser l'autre-technique qui n'est pas, c'est-à-dire le milieu associé de la pierre. En paraphrasant l'auteur, on pourrait dire que, étant à la fois carrefour et synthèse de l'archéologie préhistorique, l'objet lithique interpelle par son milieu associé à jamais manquant. Ici, le concept de "mémoire dérobée ", introduit par l'auteur, est particulièrement important et trouvera, nous l'espérons, une suite. La pierre nous a volé la mémoire du végétal. La pierre, à plusieurs reprises, nous prive de la mémoire du milieu associé préhistorique. La recherche de H. Forestier tente de nous rendre la mémoire du végétal, de l'éphémère, du périssable, permettant ainsi de passer de l'artéfact à l'individu technique, et dans ce sens, de révéler l'altérité forestière cachée sous l'ombre de la pierre.

Bref, La pierre et son ombre est un livre bienvenu car attendu puisqu'il nous offre les clés d'une anthropologie des techniques orientée vers l'éphémère. Une anthropologie des techniques éphémères.

\section{Références}

Audouze, F. 1999, New advances in French prehistory. Antiquity, 73(279): 167-175. doi:10.1017/s0003598x00087986

de Beaune, S. 2007, La préhistoire est-elle toujours une science humaine? In: ...Aux conceptions d'aujourd'hui vol. III, (Évin, J., Ed.), Un siècle de construction du discours scientifique en Préhistoire. Actes du Congrès Préhistorique de France, XXVIe session, Congrès du Centenaire, 21-25 septembre 2004, Avignon, Mémoires de la Société préhistorique française, Paris: p. 13-21.

Boëda, E. 2013, Techno-logique \& Technologie. Une Paléo-histoire des objets lithiques tranchants. @rchéo-éditions, Prigonrieux, 264 p.

Cataldi, M. 2016, Faire “parler” les pierres. Le modèle naturaliste en archéologie préhistorique. Le cas de la Vallée des Merveilles, 1868-1902. In: Signes et communication dans les civilisations sans paroles (Buchsenschutz, O., Jeunesse, C., Mordant, C., et Vialou, D., Eds.), Actes du 139e congrès du CTHS, 2016, Éditions du Comité des travaux historiques et scientifiques, Paris, p. 113-126.

Hussain, S. 2019, The French-Anglophone divide in lithic research: A plea for pluralism in Palaeolithic archaeology. Ph. D Thesis at the Faculty of Archaeology, Leiden University, Leiden, 470 p. 
Lowther, G. 1962, Epistemology and Archaeological Theory. Current Anthropology, 3(5): 495-509. URL: http://www.jstor.org/stable/2739695

Trigger, B. G. 2007, A History of Archaeological Thought. Second Edition. Cambridge: Cambridge University Press. doi:10.1017/CBO9780511813016

Simondon, G. 2012, Du mode d'existence des objets techniques (5e ed.). Aubier, Paris, 367 p.

Simondon, G. 2013, L'individuation à la lumière des notions de forme et d'information (2e ed.). Éditions Jérôme Millon, Grenoble, 563 p. 